\title{
An Analysis of Trends, Growth and Changing Pattern of Public Expenditure on Higher Education in India
}

\author{
Mr. Pulak Bhattacharyya ${ }^{1}$, Dr. (Mrs.) Manashee Gogoi ${ }^{2}$ \\ ${ }^{1}$ Assistant Professor, Department of Education, Women's College, Tinsukia, Assam, India \\ ${ }^{2}$ Associate Professor \& HOD, Department of Education, Dibrugarh University, Dibrugarh, Assam, India
}

\begin{abstract}
Higher education plays an important role in the creation of human capital. After independence India made significant improvement in the field of education including higher education. Growth of higher educational institution as well as enrolment in higher education has increased during the postindependence era. Government expenditure on higher education increased tremendously and it helped in the expansion of this sector. But, despite the massive increase in number of higher educational institution, the percentage of enrollment is not very high. Because, higher education is still not accessible to the poorest section of the population due to high cost associated with it. In this paper an attempt has been made to analyse the trend, growth and changing pattern of higher education in India.
\end{abstract}

\section{KEY WORDS: Public expenditure, Higher} Education, Compound annual growth rate

\subsection{INTRODUCTION}

Education is an important component of social sector and a pivotal factor of economic development. It is one of the most empowering tools for an individual. For overall development of a country education is very much important. It helps in economic progress as well as social progress of a country. From economic point of view, it provides the economy with requisite qualification and skilled manpower that can use its available resources properly. From society's point of view education creates awareness of social realities in the minds of the people and it helps them to attain a better standard of living. Education is considered as public good and it is included in the concurrent list of Indian constitution. Therefore, both central and state government has the responsibility to maintain quantity, quality, access and equity in education (Araf, 2016). Public expenditure on education is an important policy instrument for realizing the education goals and for the development of education. Public expenditure refers to the expenses incurred by the government for its own maintenance as also for the preservation and welfare of society and the economy as a whole (Chand, 2013). Educational expenditure constitutes an important part of the total public expenditure in India. The National Policy on Education 1986 visualises education on different phases (Pandey, 2016) and among these phases role of higher education is more meaningful in the context of social development. Higher education is defined as the education obtained after completing 12 years of schooling or equivalent and is of the duration of at least nine months (full time) or after completing 10 years of schooling and is of the duration of at least 3 years. The education may be of the nature of General, Vocational, Professional or Technical education (MHRD, 2011). In this paper, an attempt has been made to analyse the trend, growth and changing pattern of higher education in India.

\subsection{Review of Literature:}

Chakrabarti and Joglekar (2006) examined patterns and changes in the allocation of government funds for education, particularly higher education, over a span of two decades using a panel of 15 major states from India. The study found that state real per capita income significantly enhances educational expenditure at the aggregate, elementary, secondary and higher levels. The researcher argued that even after controlling for the economic reform process, privatization exerts a negative significant impact on expenditure on higher education.

Mitra (2015) made an attempt to build an empirical ground for supporting the increased role of public funding in higher education in a research work 
entitled "Public Spending in Higher Education in India: A Benefit Incidence Analysis". In this research work the trend of per capita public expenditure on higher education have been calculated from 1990-91 to 2009-10 using 2004-05 constant price. Various tools like benefit incidence analysis, suits index and concentration curves have been used by the researcher to fulfill the objectives of the study and found a prorich distribution of subsidy at the higher level of education at all India level.

Pandey (2016) in a study mentioned that the plan expenditure on education witnessed the increasing trend throughout the time period from 1990-91 to 201-12 whereas non-plan expenditure was highly incurred than the plan expenditure.

Araf (2016) in a research work examined the level, trends, growth and intra-sectoral allocation of public expenditure on education. This study found that quantum of expenditure on education has increased significantly since 2001 . The study also revealed that percentage share of state government has declined and share of central government has increased.

\subsection{Objectives of the Study:}

The objective of this paper is to analyse the trends, growth and changing pattern of public expenditure on higher education in India. The specific objectives are-

1.3.1 To analyse the trends and pattern of public expenditure on education in India.

1.3.2 To analyse the growth trend of higher education in India.

1.3.3 To investigate the growth trend of higher education in Assam.

\subsection{Methodology:}

This paper is entirely based on secondary data. Data has been collected from various reports published by
Ministry of Human Resource Development (MHRD) and Reserve Bank of India. To show the trends, growth and changing pattern of public expenditure data has been used for the period 2000-01 to2011-12 while the changing scenario of higher education is shown by using the data from 2001-02 to 2017-18. Information has also been collected from various books, journals, theses and periodicals for the purpose of the study.

The study is descriptive and analytical in nature. To show the growth trend of public expenditure on higher education and growth trend of higher education system, compound annual growth rate (CAGR), annual growth rate has been calculated.

\subsection{Analysis and Findings:}

\subsubsection{Public Expenditure on Education in India:}

Education is an important component of social sector and both state government and the central government have the responsibility of it. Though, the state government has to take the major responsibilities of its education sector, but, some responsibilities lie within the jurisdiction of central government. These are mainly coordination of educational facilities, determination of standards of higher education and scientific and technical education. The $42^{\text {nd }}$ amendment of the constitution of India in 1976, brought education from the "state list" to "concurrent list", whereby central government gained more power to intervene in education at all levels (Pandey, 2016).

The total (state and central government) educational expenditure expressed as a percentage of GDP gives a good indication of priority given to education in society (Chakrabarti and Joglekar, 2006). The table 1 shows the trend of public expenditure on education by state and central government as well as public expenditure as a percentage of GDP.

Table 1: Trend of Public Expenditure on Education in India

\begin{tabular}{|c|c|c|c|c|c|c|c|c|}
\hline \multirow[t]{2}{*}{ Years } & \multicolumn{2}{|c|}{$\begin{array}{l}\text { Expenditure on } \\
\text { Education (Rs. In } \\
\text { crore) }\end{array}$} & \multirow[t]{2}{*}{ Total } & \multirow[t]{2}{*}{$\begin{array}{c}\text { Growth } \\
\text { Rate }\end{array}$} & \multirow[t]{2}{*}{$\begin{array}{l}\% \text { share } \\
\text { of centre }\end{array}$} & \multirow[t]{2}{*}{$\begin{array}{l}\% \text { share } \\
\text { of state }\end{array}$} & \multirow{2}{*}{$\begin{array}{l}\text { State } \\
\text { as } \% \\
\text { of GDP }\end{array}$} & \multirow{2}{*}{$\begin{array}{c}\text { State + } \\
\text { Centre as } \\
\% \text { of } \\
\text { GDP }\end{array}$} \\
\hline & Centre & State & & & & & & \\
\hline $\begin{array}{l}2000- \\
2001\end{array}$ & 10195.95 & 72290.53 & 82486.48 & - & 12.36075 & 87.63925 & 3.63 & 4.14 \\
\hline $\begin{array}{l}2001- \\
2002 \\
\end{array}$ & 14119.52 & 65746.19 & 79865.71 & -3.17 & 17.67908 & 82.32092 & 3.03 & 3.68 \\
\hline $\begin{array}{l}2002- \\
2003\end{array}$ & 16156.63 & 69350.70 & 85507.33 & 7.06 & 18.89502 & 81.10498 & 2.97 & 3.66 \\
\hline
\end{tabular}


International Journal of Trend in Scientific Research and Development (IJTSRD) ISSN: 2456-6470

\begin{tabular}{|c|c|c|c|c|c|c|c|c|}
\hline $\begin{array}{l}2003- \\
2004\end{array}$ & 17100.97 & 71978.28 & 89079.25 & 4.18 & 19.19748 & 80.80252 & 2.74 & 3.40 \\
\hline $\begin{array}{l}2004- \\
2005\end{array}$ & 18025.96 & 78668.14 & 96694.10 & 8.54 & 18.64225 & 81.35775 & 2.65 & 3.26 \\
\hline $\begin{array}{l}2005- \\
2006\end{array}$ & 23209.77 & 90018.94 & 113228.71 & 17.09 & 20.49813 & 79.50187 & 2.66 & 3.34 \\
\hline $\begin{array}{l}2006- \\
2007\end{array}$ & 34236.52 & 103147.47 & 137383.99 & 21.33 & 24.92031 & 75.07969 & 2.61 & 3.48 \\
\hline $\begin{array}{l}2007- \\
2008\end{array}$ & 39919.37 & 115877.90 & 155797.27 & 13.40 & 25.62264 & 74.37736 & 2.53 & 3.40 \\
\hline $\begin{array}{l}2008- \\
2009\end{array}$ & 47977.59 & 141091.25 & 189068.84 & 21.36 & 25.37573 & 74.62427 & 2.66 & 3.56 \\
\hline $\begin{array}{l}2009- \\
2010\end{array}$ & 64023.23 & 177232.79 & 241256.02 & 27.60 & 26.53746 & 73.46254 & 2.90 & 3.95 \\
\hline $\begin{array}{l}2010- \\
2011\end{array}$ & 80660.73 & 212817.50 & 293478.23 & 21.64 & 27.4844 & 72.5156 & 2.94 & 4.05 \\
\hline $\begin{array}{l}2011- \\
2012\end{array}$ & 86074.52 & 247855.86 & 333930.38 & & 25.77619 & 74.22381 & 2.84 & 3.82 \\
\hline $\begin{array}{l}2012- \\
2013\end{array}$ & 89757.60 & 278375.27 & 368132.87 & 10.24 & 24.38185 & 75.61815 & 2.80 & 3.70 \\
\hline $\begin{array}{l}2013- \\
2014 \mathrm{RE})\end{array}$ & 101594.26 & 332046.33 & 433640.59 & 17.79 & 23.42822 & 76.57178 & 2.96 & 3.86 \\
\hline $\begin{array}{l}2014- \\
2015(\mathrm{BE})\end{array}$ & 122489.34 & 380440.01 & 502929.35 & 15.98 & 24.35518 & 75.64482 & 3.06 & 4.04 \\
\hline CAGR & 19.01 & 12.33 & 13.49 & in -5 & entfic & -0 & - & - \\
\hline
\end{tabular}

RE- Revised Estimate BE- Budget Estimate

Source: Analysis of Budgeted Expenditure on Education by MHRD

Table 1 show that public expenditure on education has increased significantly during the time 2000-01 to 201415. Expenditure on education incurred by central government has registered a compound annual growth rate of $19.01 \%$ while expenditure by state government has registered a growth rate of $12.33 \%$. On the other hand, total expenditure (both central and state government) shows a growth rate of 13.49\% during the time period 2000-01 to 2014-15. It is also seen from the table that the share of state government in total expenditure has decreased from $87.64 \%$ in $2000-01$ to $75.64 \%$ in $2014-15$. The share of central government has increased from $12.36 \%$ to $24.36 \%$ during the same period. If we analyse the government expenditure as percentage of GDP we see that it was $4.14 \%$ in 2000-01. But public expenditure as percentage of GDP has decreased from 4.14\% in $2000-01$ to $4.04 \%$ in $2014-15$.

\subsubsection{Public Expenditure on Education- As ratio to Aggregate Expenditure:}

To assess the Government's emphasis on education, it is very essential to analyse the trend of public expenditure allocated as well as incurred by the Government in the field of education. The table 2 shows the priority given to education sector in North- Eastern states and in India as a whole.

Table 2: Expenditure on Education - As ratio to Aggregate Expenditure of North East Indiia

\begin{tabular}{|c|c|c|c|c|c|c|c|c|c|c|c|c|c|c|c|c|c|}
\hline $\mathbf{S}$ & $\begin{array}{c}200 \\
1- \\
02\end{array}$ & $\begin{array}{c}200 \\
2- \\
03\end{array}$ & $\begin{array}{c}200 \\
3- \\
04\end{array}$ & $\begin{array}{c}200 \\
4- \\
05\end{array}$ & $\begin{array}{c}200 \\
5- \\
06\end{array}$ & $\begin{array}{c}200 \\
6- \\
07\end{array}$ & $\begin{array}{c}200 \\
7- \\
08\end{array}$ & $\begin{array}{c}200 \\
8- \\
09\end{array}$ & $\begin{array}{c}200 \\
9- \\
10\end{array}$ & $\begin{array}{c}201 \\
0- \\
11\end{array}$ & $\begin{array}{c}201 \\
1- \\
12\end{array}$ & $\begin{array}{c}201 \\
2- \\
13\end{array}$ & $\begin{array}{c}201 \\
3- \\
14\end{array}$ & $\begin{array}{c}201 \\
4- \\
15\end{array}$ & $\begin{array}{c}201 \\
5- \\
16\end{array}$ & $\begin{array}{c}201 \\
6- \\
17 \\
(\mathrm{R} \\
\mathrm{E})\end{array}$ & $\begin{array}{c}201 \\
7- \\
18 \\
\text { (B } \\
\text { E) }\end{array}$ \\
\hline $\begin{array}{l}\text { Arunac } \\
\text { hal } \\
\text { pradesh }\end{array}$ & $\begin{array}{c}13 . \\
3\end{array}$ & $\begin{array}{c}12 \\
1\end{array}$ & 9.1 & $\begin{array}{c}10 . \\
7\end{array}$ & 9.9 & $\begin{array}{c}10 . \\
7\end{array}$ & $\begin{array}{c}10 . \\
8\end{array}$ & $\begin{array}{c}11 \\
4\end{array}$ & $\begin{array}{c}12 . \\
2\end{array}$ & $\begin{array}{c}10 . \\
8\end{array}$ & $\begin{array}{c}11 . \\
4\end{array}$ & $\begin{array}{c}12 . \\
2\end{array}$ & $\begin{array}{c}11 . \\
5\end{array}$ & $\begin{array}{c}13 . \\
2\end{array}$ & $\begin{array}{c}11 . \\
6\end{array}$ & $\begin{array}{c}10 \\
4\end{array}$ & $\begin{array}{c}10 . \\
9\end{array}$ \\
\hline
\end{tabular}


International Journal of Trend in Scientific Research and Development (IJTSRD) ISSN: 2456-6470

\begin{tabular}{|c|c|c|c|c|c|c|c|c|c|c|c|c|c|c|c|c|c|}
\hline Assam & $\begin{array}{c}21 . \\
9\end{array}$ & $\begin{array}{c}22 . \\
4\end{array}$ & $\begin{array}{c}22 . \\
3\end{array}$ & $\begin{array}{c}17 . \\
0\end{array}$ & $\begin{array}{c}20 . \\
8\end{array}$ & $\begin{array}{c}20 . \\
4\end{array}$ & $\begin{array}{c}20 . \\
1\end{array}$ & $\begin{array}{c}18 . \\
8\end{array}$ & $\begin{array}{c}16 . \\
4\end{array}$ & $\begin{array}{c}22 . \\
0\end{array}$ & $\begin{array}{c}20 . \\
3\end{array}$ & $\begin{array}{c}20 . \\
6\end{array}$ & $\begin{array}{c}22 . \\
6\end{array}$ & $\begin{array}{c}24 . \\
7\end{array}$ & $\begin{array}{c}25 . \\
5\end{array}$ & $\begin{array}{c}20 . \\
2\end{array}$ & $\begin{array}{c}18 . \\
3\end{array}$ \\
\hline $\begin{array}{c}\text { Manip } \\
\text { ur }\end{array}$ & $\begin{array}{c}13 . \\
7\end{array}$ & $\begin{array}{c}13 . \\
3\end{array}$ & $\begin{array}{c}13 . \\
1\end{array}$ & $\begin{array}{c}15 . \\
3\end{array}$ & $\begin{array}{c}15 . \\
4\end{array}$ & $\begin{array}{c}11 . \\
9\end{array}$ & $\begin{array}{c}14 . \\
2\end{array}$ & $\begin{array}{c}12 . \\
0\end{array}$ & $\begin{array}{c}11 . \\
9\end{array}$ & $\begin{array}{c}10 . \\
7\end{array}$ & $\begin{array}{c}10 . \\
6\end{array}$ & $\begin{array}{c}11 . \\
7\end{array}$ & $\begin{array}{c}12 . \\
8\end{array}$ & $\begin{array}{c}14 . \\
0\end{array}$ & $\begin{array}{c}12 . \\
5\end{array}$ & $\begin{array}{c}11 . \\
8\end{array}$ & $\begin{array}{c}13 . \\
1\end{array}$ \\
\hline $\begin{array}{c}\text { Meghal } \\
\text { aya }\end{array}$ & $\begin{array}{c}17 . \\
9\end{array}$ & $\begin{array}{c}15 . \\
3\end{array}$ & $\begin{array}{c}15 . \\
2\end{array}$ & $\begin{array}{c}15 . \\
0\end{array}$ & $\begin{array}{c}15 . \\
5\end{array}$ & $\begin{array}{c}14 . \\
1\end{array}$ & $\begin{array}{c}15 . \\
5\end{array}$ & $\begin{array}{c}12 . \\
8\end{array}$ & $\begin{array}{c}14 . \\
8\end{array}$ & $\begin{array}{c}16 . \\
1\end{array}$ & $\begin{array}{c}17 . \\
3\end{array}$ & $\begin{array}{c}15 . \\
8\end{array}$ & $\begin{array}{c}16 . \\
6\end{array}$ & $\begin{array}{c}17 . \\
1\end{array}$ & $\begin{array}{c}16 . \\
2\end{array}$ & $\begin{array}{c}15 . \\
3\end{array}$ & $\begin{array}{c}15 . \\
9\end{array}$ \\
\hline $\begin{array}{c}\text { Mizora } \\
\mathrm{m}\end{array}$ & $\begin{array}{c}16 . \\
0\end{array}$ & $\begin{array}{c}14 . \\
5\end{array}$ & $\begin{array}{c}12 . \\
0\end{array}$ & $\begin{array}{c}13 . \\
8\end{array}$ & $\begin{array}{c}13 . \\
4\end{array}$ & $\begin{array}{c}13 . \\
8\end{array}$ & $\begin{array}{c}13 . \\
2\end{array}$ & $\begin{array}{c}14 . \\
1\end{array}$ & $\begin{array}{c}14 . \\
9\end{array}$ & $\begin{array}{c}14 . \\
9\end{array}$ & $\begin{array}{c}15 . \\
7\end{array}$ & $\begin{array}{c}15 . \\
7\end{array}$ & $\begin{array}{c}17 . \\
1\end{array}$ & $\begin{array}{c}17 . \\
4\end{array}$ & $\begin{array}{c}17 . \\
6\end{array}$ & $\begin{array}{c}13 . \\
2\end{array}$ & $\begin{array}{c}14 . \\
0\end{array}$ \\
\hline $\begin{array}{l}\text { Nagala } \\
\text { nd }\end{array}$ & $\begin{array}{c}11 . \\
0\end{array}$ & $\begin{array}{c}11 . \\
0\end{array}$ & $\begin{array}{c}10 . \\
8\end{array}$ & $\begin{array}{c}11 . \\
0\end{array}$ & $\begin{array}{c}11 . \\
6\end{array}$ & $\begin{array}{c}12 . \\
3\end{array}$ & $\begin{array}{c}11 . \\
4\end{array}$ & $\begin{array}{c}11 . \\
2\end{array}$ & $\begin{array}{c}11 . \\
3\end{array}$ & $\begin{array}{c}13 . \\
4\end{array}$ & $\begin{array}{c}12 . \\
1\end{array}$ & $\begin{array}{c}13 . \\
2\end{array}$ & $\begin{array}{c}15 . \\
3\end{array}$ & $\begin{array}{c}13 . \\
7\end{array}$ & $\begin{array}{c}14 . \\
0\end{array}$ & $\begin{array}{c}14 . \\
6\end{array}$ & $\begin{array}{c}14 . \\
7\end{array}$ \\
\hline Sikkim & 8.0 & 7.6 & $\begin{array}{c}11 . \\
8\end{array}$ & 8.8 & $\begin{array}{c}10 . \\
4\end{array}$ & $\begin{array}{c}10 . \\
5\end{array}$ & 9.2 & $\begin{array}{c}10 . \\
6\end{array}$ & $\begin{array}{c}12 . \\
4\end{array}$ & $\begin{array}{c}17 . \\
3\end{array}$ & $\begin{array}{c}14 . \\
0\end{array}$ & $\begin{array}{c}15 . \\
0\end{array}$ & $\begin{array}{c}15 . \\
6\end{array}$ & $\begin{array}{c}15 . \\
8\end{array}$ & $\begin{array}{c}17 . \\
6\end{array}$ & $\begin{array}{c}16 . \\
6\end{array}$ & $\begin{array}{c}16 . \\
4\end{array}$ \\
\hline Tripura & $\begin{array}{c}18 . \\
6\end{array}$ & $\begin{array}{c}19 . \\
2\end{array}$ & $\begin{array}{c}18 . \\
3\end{array}$ & $\begin{array}{c}20 . \\
0\end{array}$ & $\begin{array}{c}15 . \\
3\end{array}$ & $\begin{array}{c}15 . \\
9\end{array}$ & $\begin{array}{c}15 . \\
1\end{array}$ & $\begin{array}{c}14 . \\
4\end{array}$ & $\begin{array}{c}16 . \\
2\end{array}$ & $\begin{array}{c}17 . \\
2\end{array}$ & $\begin{array}{c}17 . \\
0\end{array}$ & $\begin{array}{c}15 . \\
9\end{array}$ & $\begin{array}{c}16 . \\
4\end{array}$ & $\begin{array}{c}15 . \\
5\end{array}$ & $\begin{array}{c}15 . \\
5\end{array}$ & $\begin{array}{c}15 . \\
8\end{array}$ & $\begin{array}{c}15 . \\
7\end{array}$ \\
\hline $\begin{array}{c}\text { All } \\
\text { States }\end{array}$ & $\begin{array}{c}16 . \\
2\end{array}$ & $\begin{array}{c}15 . \\
1\end{array}$ & $\begin{array}{c}12 . \\
6\end{array}$ & $\begin{array}{c}12 . \\
7\end{array}$ & $\begin{array}{c}14 . \\
2\end{array}$ & $\begin{array}{c}14 . \\
0\end{array}$ & $\frac{13 .}{8}$ & $\begin{array}{c}14 . \\
3\end{array}$ & $\frac{15 .}{3}$ & $\begin{array}{r}16 . \\
6\end{array}$ & $\begin{array}{c}16 . \\
3\end{array}$ & $\begin{array}{c}16 . \\
4\end{array}$ & $\begin{array}{c}16 . \\
5\end{array}$ & $\begin{array}{c}16 . \\
0\end{array}$ & $\begin{array}{c}15 . \\
3\end{array}$ & $\begin{array}{c}14 . \\
8\end{array}$ & $\begin{array}{c}14 . \\
8\end{array}$ \\
\hline $\begin{array}{c}\text { All } \\
\text { States } \\
\text { (perce } \\
\text { nt to } \\
\text { GDP) }\end{array}$ & 2.6 & 2.5 & 2.3 & 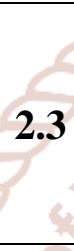 & 2 & 2.2 & 2.2 & 2.3 & 2.4 & 2.5 & 2.5 & 2.5 & 2.5 & 2.6 & 2.6 & 2.8 & 2.8 \\
\hline
\end{tabular}

RE: Revised Estimates BE: Budget Estimates (In percent)

Source: State Finances, A Study of Budgets of 2017-18 and 2018-19, RBI

It is seen from table 2 that during 2001-02 India incurred 16.2 percent of its total expenditure on education sector. State wise analysis shows that in the states of Assam, Meghalaya and Tripura percentage spent on education is higher than the national level. In this time period, among the North-Eastern states Assam incurred highest $(21.9 \%)$ of its total expenditure on education while Sikkim incurred lowest $(8.0 \%)$. Time series analysis of ratio expenditure on education to aggregate expenditure by the North-Eastern states during the period 200102 to 2017-18 exhibits a fluctuating trend. Table shows that share of educational expenditure in total government expenditure has declined between 2001-02and 2017-18 in the states of Arunachal Pradesh, Assam, Manipur, Meghalaya, Mizoram and Tripura. National level data also shows a decline during this time period. Only in the states of Nagaland and Sikkim share of educational expenditure to total government expenditure has shown an increase. In the states of Sikkim percentage of educational expenditure to total public expenditure has shown a two times increase from 8 percent in 2001-02 to 16.4 percent in 2017-18.

\subsubsection{Public Expenditure on Higher Education in India:}

Higher education can be considered as a public good since it has the characteristic of externality. A huge set of externalities namely, economic, social, cultural, demographic and political externalities are associated with higher education. It can also be considered as merit good, since the Ministry of Finance, Government of India, has recognized post-elementary education at least as a Merit-2 good. Higher education is regarded crucial to the development of developing countries and to their ability to compete in the global economy. As reported by the Punnayya Committee on Higher Education (1993), higher education determines India's economic and technological progress, and government funding must continue to be an essential and mandatory requirement for support to higher education.

The expenditure incurred by the government on higher education can be classified into two categories. One is plan expenditure and another is non-plan expenditure. Plan expenditure refers to the amount spent on the recruitment of new staff, construction of universities and colleges etc. On the other hand, Non-Plan expenditure is incurred on the maintenance of established infrastructure and committed expenditures. The table 3 shows the plan and non-plan expenditure incurred by the state government on higher education. 
International Journal of Trend in Scientific Research and Development (IJTSRD) ISSN: 2456-6470

Table 3: State Government Expenditure on Higher Education in India

\begin{tabular}{|c|c|c|c|c|c|}
\hline Year & $\begin{array}{c}\text { Plan Expenditure } \\
\text { on Higher } \\
\text { Education }\end{array}$ & $\begin{array}{c}\text { Percentage of } \\
\text { Plan } \\
\text { Expenditure }\end{array}$ & $\begin{array}{c}\text { Non-Plan } \\
\text { Expenditure on } \\
\text { Higher Education }\end{array}$ & $\begin{array}{c}\text { Percentage of } \\
\text { Non-Plan } \\
\text { Expenditure }\end{array}$ & $\begin{array}{c}\text { Total } \\
\text { Expenditure }\end{array}$ \\
\hline $2000-01$ & 347.9 & 5.35 & 6561.4 & 94.96 & 6909.4 \\
\hline $2001-02$ & 421.7 & 6.55 & 6018.2 & 93.45 & 6440.0 \\
\hline $2002-03$ & 431.2 & 6.07 & 6676.5 & 93.93 & 7107.7 \\
\hline $2003-04$ & 410.3 & 5.62 & 6888.2 & 94.38 & 7298.5 \\
\hline $2004-05$ & 494.4 & 6.68 & 6909.7 & 93.32 & 7404.2 \\
\hline $2005-06$ & 744.3 & 8.57 & 7937.5 & 91.43 & 8681.9 \\
\hline $2006-07$ & 904.6 & 9.44 & 8680.8 & 90.56 & 9585.4 \\
\hline $2007-08$ & 1152.6 & 11.07 & 9263.3 & 88.93 & 10416.0 \\
\hline $2008-09$ & 1276.2 & 10.55 & 10822.4 & 89.45 & 12098.6 \\
\hline $2009-10$ & 1590.6 & 10.4 & 14248.8 & 89.96 & 15939.4 \\
\hline $2010-11$ & 1610.2 & 11.04 & 15420.7 & 89.92 & 17131.86 \\
\hline $2011-12$ & 1705.7 & 12.17 & 17535.8 & 91.04 & 19344.71 \\
\hline CAGR & $\mathbf{1 3 . 5 6}$ & - & $\mathbf{8 . 1 8}$ & - & $\mathbf{8 . 5 9}$ \\
\hline
\end{tabular}

Source: Annual Reports, Analysis of Budgeted Expenditure on Education Various Issues

It is seen from table 3 that the plan expenditure on higher education incurred by the state government has increased at a compound annual growth rate of $13.56 \%$ during the time period 2000-01 to 2011-12. As against this, the non-plan expenditure of the state government on higher education has registered a growth rate of $8.18 \%$ during the same time period. Total expenditure (both plan and non-plan) has increased at a growth rate of $8.59 \%$. If we see the percentage of plan and non-plan expenditure, plan expenditure shows an increasing trend while non-plan expenditure shows a fluctuating during 2000-01 to 2011-12. The table also shows that the non-plan expenditure was comparatively higher in comparison to plan expenditure during this time period.

Table 4: Central Government Expenditure on Higher Education:

\begin{tabular}{|c|c|c|c|c|c|}
\hline Year & $\begin{array}{c}\text { Plan Expenditure } \\
\text { on Higher } \\
\text { Education }\end{array}$ & $\begin{array}{c}\text { Percentage of } \\
\text { Plan Expenditure }\end{array}$ & $\begin{array}{c}\text { Non-Plan } \\
\text { Expenditure on } \\
\text { Higher Education }\end{array}$ & $\begin{array}{c}\text { Percentage of } \\
\text { Non-Plan } \\
\text { Expenditure }\end{array}$ & $\begin{array}{c}\text { Total } \\
\text { Expenditure }\end{array}$ \\
\hline $2000-01$ & 497.5 & 21.77 & 1787.7 & 78.23 & 2285.3 \\
\hline $2001-02$ & 544.7 & 33.06 & 1102.9 & 66.94 & 1647.6 \\
\hline $2002-03$ & 619.1 & 35.34 & 1132.7 & 64.66 & 1751.8 \\
\hline $2003-04$ & 560.4 & 31.81 & 1201.1 & 68.19 & 1761.5 \\
\hline $2004-05$ & 810.6 & 38.62 & 1288.3 & 61.38 & 2099.0 \\
\hline $2005-06$ & 843.5 & 36.18 & 1487.8 & 63.82 & 2331.4 \\
\hline $2006-07$ & 1353.4 & 45.79 & 1602.1 & 54.21 & 2955.5 \\
\hline $2007-08$ & 1903.1 & 48.86 & 1992.2 & 51.14 & 3895.3 \\
\hline $2008-09$ & 3684.1 & 56.62 & 2822.4 & 43.38 & 6506.4 \\
\hline $2009-10$ & 4090.1 & 49.00 & 4256.6 & 51.00 & 8346.6 \\
\hline $2010-11$ & 4180.2 & 51.07 & 4270.7 & 51.79 & 8553.76 \\
\hline $2011-12$ & 4352.5 & 53.83 & 4785.9 & 54.62 & 9246.85 \\
\hline CAGR & $\mathbf{1 8 . 9 5}$ & - & $\mathbf{8 . 2 0}$ & - & $\mathbf{1 1 . 8 3}$ \\
\hline
\end{tabular}

Source: Annual Reports, Analysis of Budgeted Expenditure, Various Years

It is seen from table 4 that the plan expenditure on higher education incurred by the central government has increased at a compound annual growth rate of $18.95 \%$ during the time period 2000-01 to 2011-12. As against this, the non-plan expenditure of the central government on higher education has registered a growth rate of $8.20 \%$ during the same time period. Total expenditure (both plan and non-plan) has increased at a growth rate of $11.83 \%$. Percentage share of plan and non-plan expenditure both shows a fluctuating trend during 2000-01 to 2011-12. 


\subsubsection{Growth Trend of Higher Education in India:}

\subsubsection{Growth in Number of Higher Educational Institutions:}

The number of higher educational institutions has increased rapidly during the time period 2000-01 to 2017-18. The number of universities has increased from 254 in 2000-01 to 903 in 2017-18 registering a compound annual growth rate of $7.91 \%$ while the number of colleges has increased from 10152 to 39050 during the same period by registering a compound annual growth rate of $8.41 \%$. This is shown in the figure 1 and 2 below.

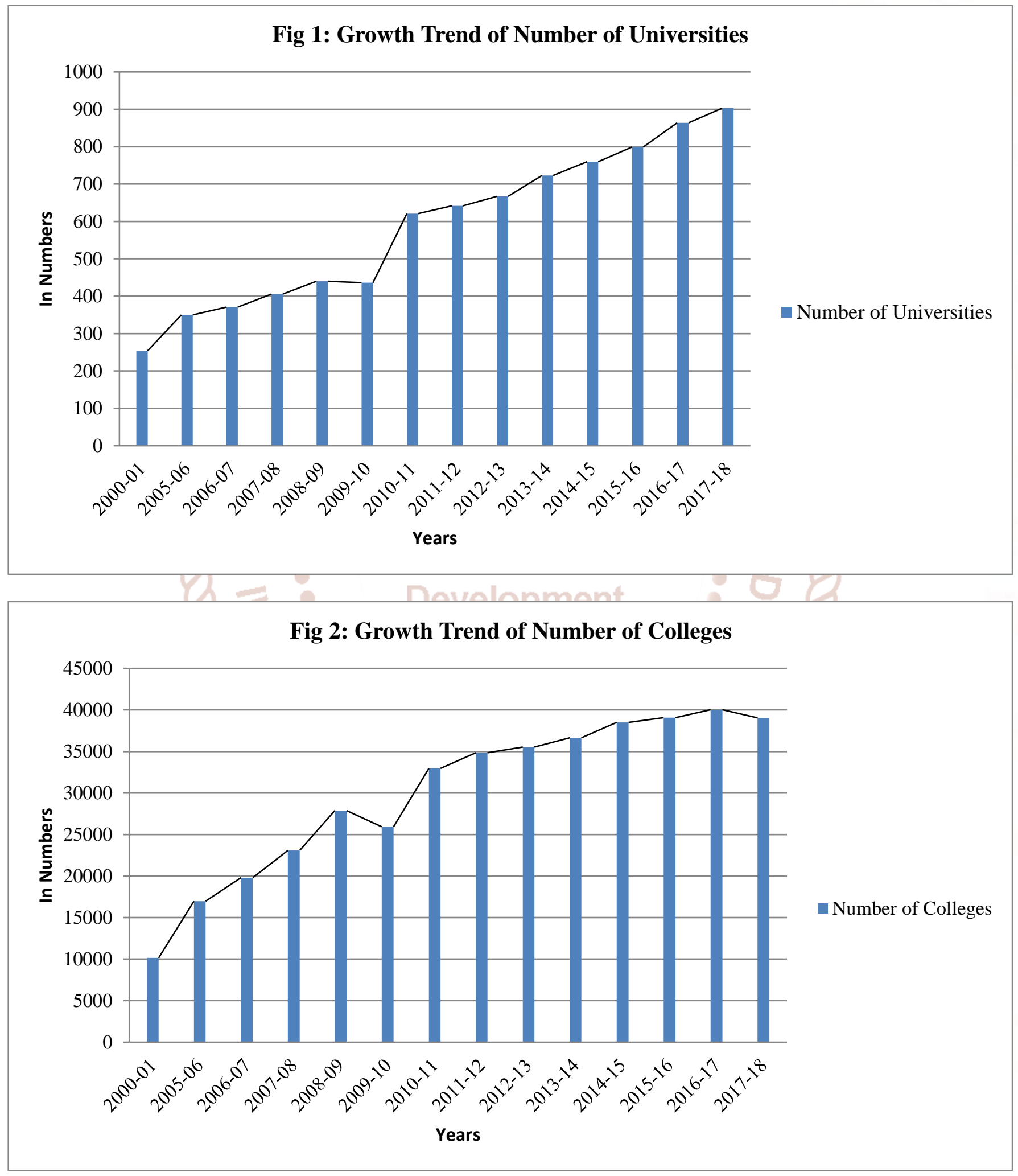


International Journal of Trend in Scientific Research and Development (IJTSRD) ISSN: 2456-6470

\subsubsection{Growth in Student Enrolment:}

Table 5: Enrolment in Higher Education from 2001-02 to 2017-18

\begin{tabular}{|c|c|c|c|}
\hline \multirow{2}{*}{ Year } & \multicolumn{2}{|c|}{ Enrolment } & \multirow{2}{*}{ Total } \\
\cline { 2 - 3 } & Male & Female & 9541826 \\
\hline $2001-02$ & 5795417 & 3746409 & 10716558 \\
\hline $2002-03$ & 6681558 & 4035000 & 11200584 \\
\hline $2003-04$ & 7044205 & 4156379 & 13032186 \\
\hline $2005-06$ & 8191957 & 4840229 & 14323566 \\
\hline $2006-07$ & 8831748 & 5491818 & 15257893 \\
\hline $2007-08$ & 9408097 & 5849796 & 17211216 \\
\hline $2008-09$ & 10573890 & 6637326 & 18500325 \\
\hline $2009-10$ & 11227810 & 7272515 & 20740740 \\
\hline $2010-11$ & 12444600 & 8296140 & 27499749 \\
\hline $2011-12$ & 15466559 & 12033190 & 30184331 \\
\hline $2012-13$ & 16173473 & 13010858 & 32336234 \\
\hline $2013-14$ & 16617294 & 13535123 & 34211637 \\
\hline $2014-15$ & 17495394 & 14840840 & 34584781 \\
\hline $2015-16$ & 18488619 & 15723018 & 35705905 \\
\hline $2016-17$ & 18594723 & 15990058 & 36642378 \\
\hline $2017-18$ & 18980595 & 16725310 & 8.41 \\
\hline CAGR & 19204675 & 17437703 & 9.67 \\
\hline
\end{tabular}

Source: (i) Data in respect of 2001-02 to 2008-09 have been taken from "Selected Educational Statistics" published by Ministry of HRD.

(ii) Data in respect of 2009-10 to 2017-18 have been taken from "All India Survey on Higher Education” published by Ministry of HRD.

Table 5 shows that the total enrolment in higher education system has increased from 9541826 in $2001-02$ to 36642378 by registering a compound annual growth rate of $8.41 \%$. For male this growth rate is $7.45 \%$ while for female it is 9.67\%. Thus, growth rate of female enrolment is higher than male enrolment in higher education system. The growth trend of total enrolment in higher education for last ten years is shown in figure 3.

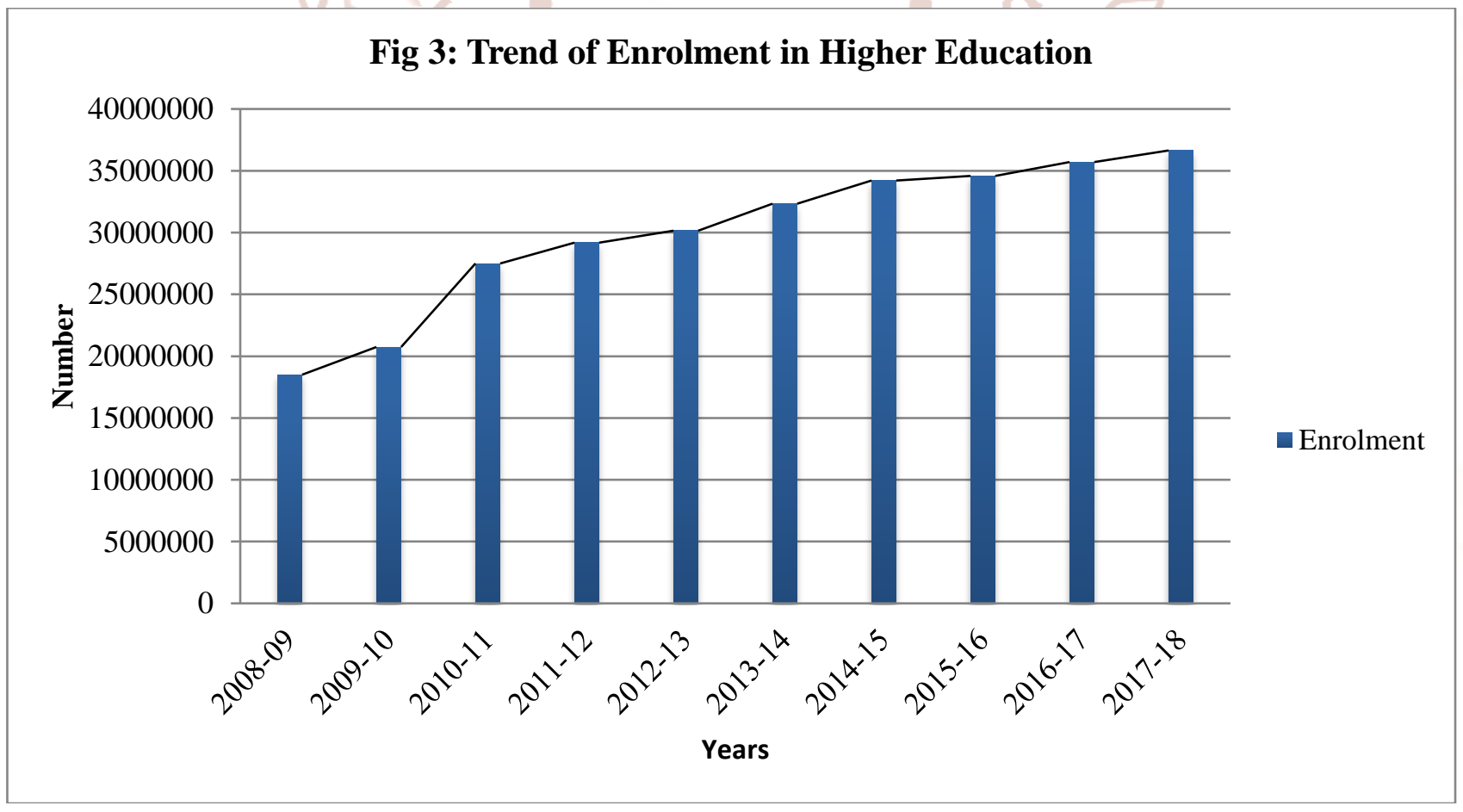




\subsubsection{Gross Enrolment Ratio in Higher Education:}

Gross Enrolment Ratio (GER) is the total enrolment in a specific level of education, regardless of age, expressed as a percentage of the eligible official age population corresponding to the same level of education in a given academic year. It is calculated by dividing the number of students enrolled in a given level of education regardless of age by the population of the age group which corresponds to the given level of education and multiplying the result by 100 (MHRD, 2011). Gross Enrolment Ratio in higher education during 2001-02 to 2017-18 is shown in the figure 4.

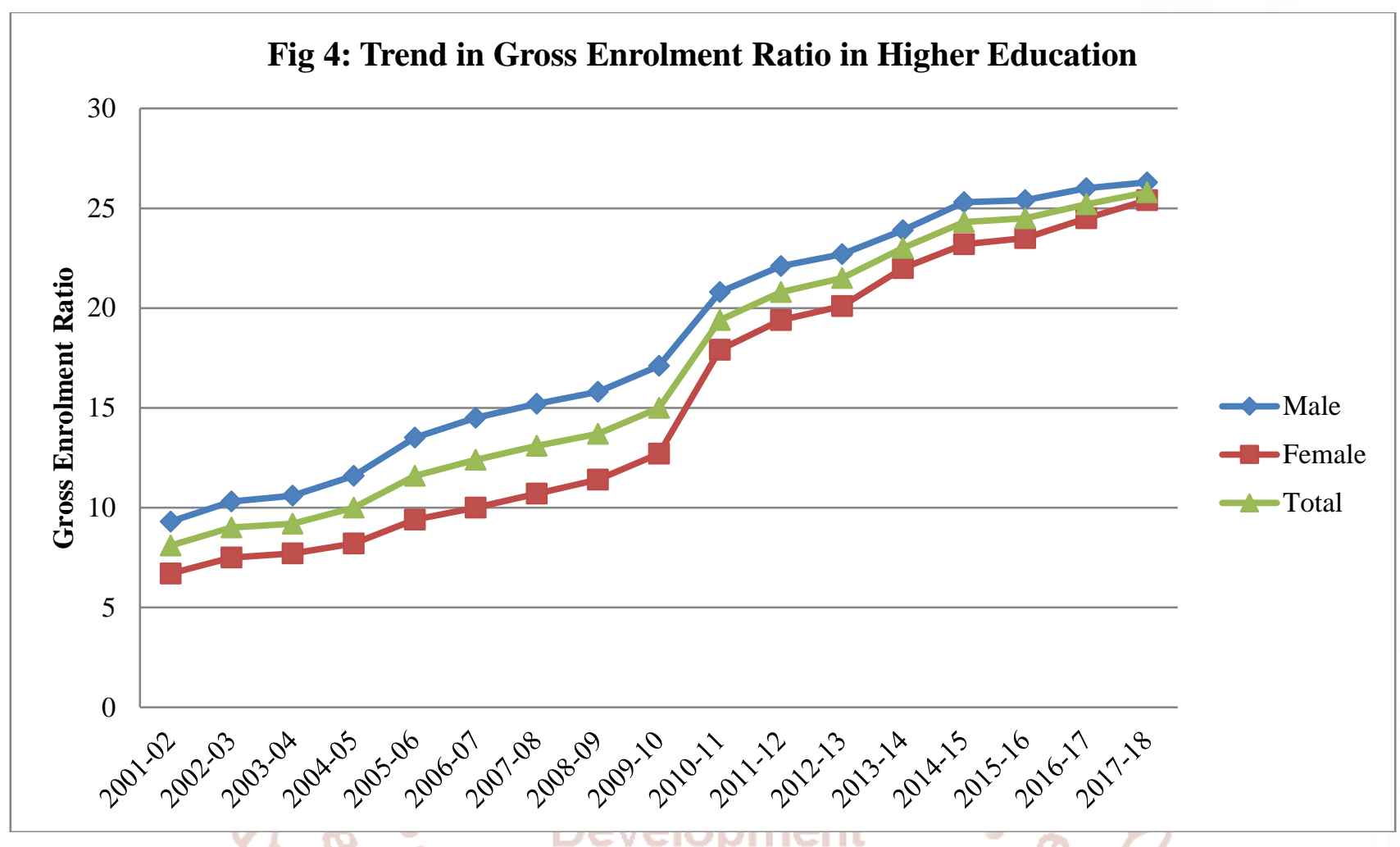

From the figure 4 it is seen that the Gross Enrolment Ratio (GER) in India has increased from 8.1\% in 2001-02 to $25.8 \%$ in 2017-18. It is clear from the figure that though, both male GER and female GER has an increasing trend during this time period, but, GER of male is higher than GER of female. The figure shows that there was a sharp increase in GER for both male and female in the year 2009-10 as a result the overall GER has also shown an increase. After that the difference between the GER of male and female has decreased and in the year 2017-18 it is found to be very negligible.

\subsubsection{Growth Trend of Higher Education in Assam:}

To analyse the growth trend of higher education in Assam this paper considers to indicators namely, student enrolment in higher education and Gross Enrolment Ratio in higher education of the state for a time period from 2010-11 to 2017-18. Later, this paper also compares the GER of Assam with all India level for this time period. Trend of student enrolment in higher education is shown in the figure 5. 


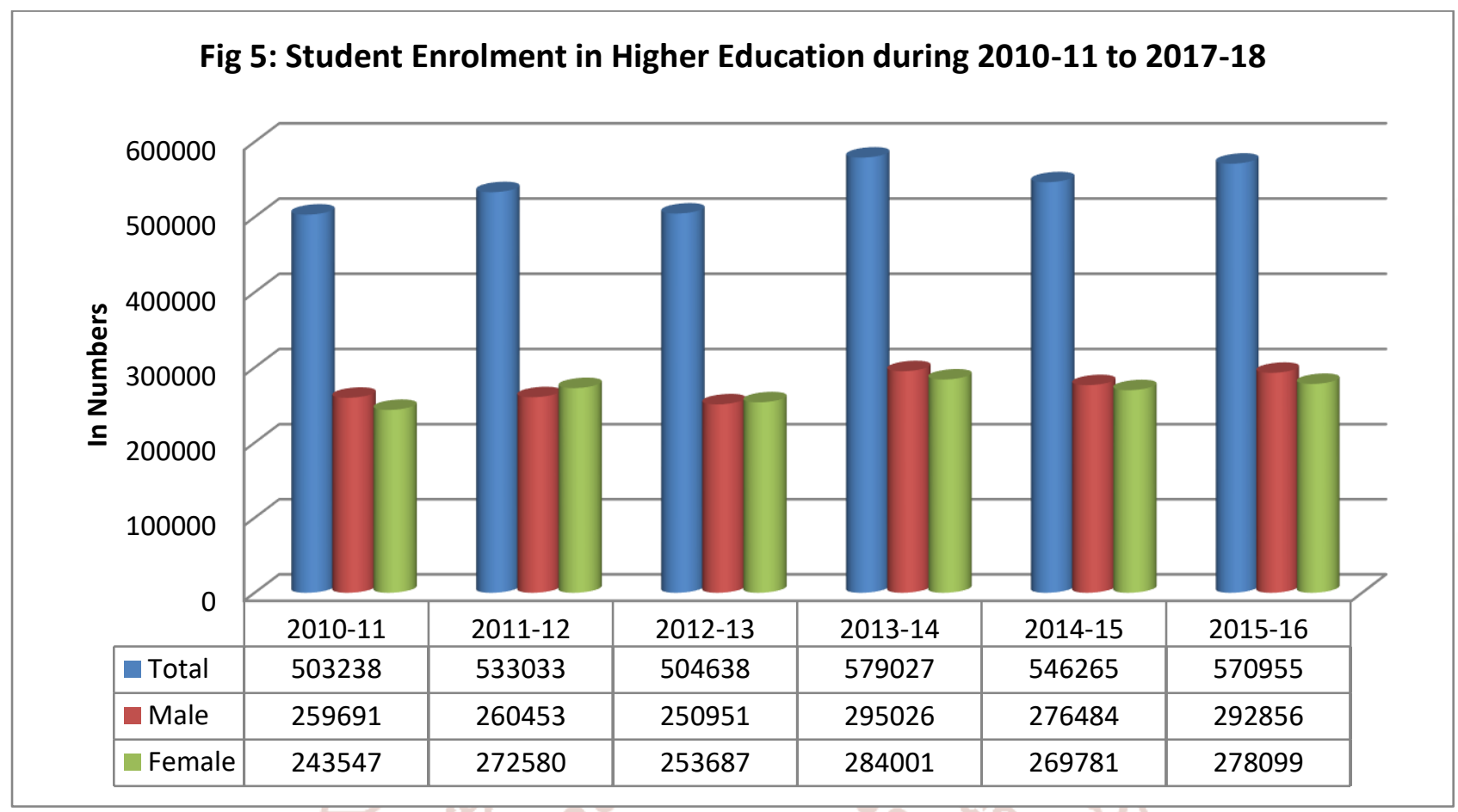

The figure 5 shows that student enrolment in higher education in Assam has increased from 503238 in 2010-11 to 678344 in $2017-18$ by registering a compound annual growth rate of $3.96 \%$. Gender wise enrolment shows that male enrolment has increased from 259691 to 336939 and female enrolment has increased from 243547 to 341405 during this stipulated period by registering compound annual growth rate of $3.44 \%$ and $4.49 \%$ respectively. Thus we see that during this period the growth rate of enrolment is not very high and female enrolment increased more rapidly than male enrolment.

\subsubsection{Gross Enrolment Ratio in Higher Education in Assam:}

Gross Enrolment Ratio in higher education in Assam is shown in the figure 6 below.

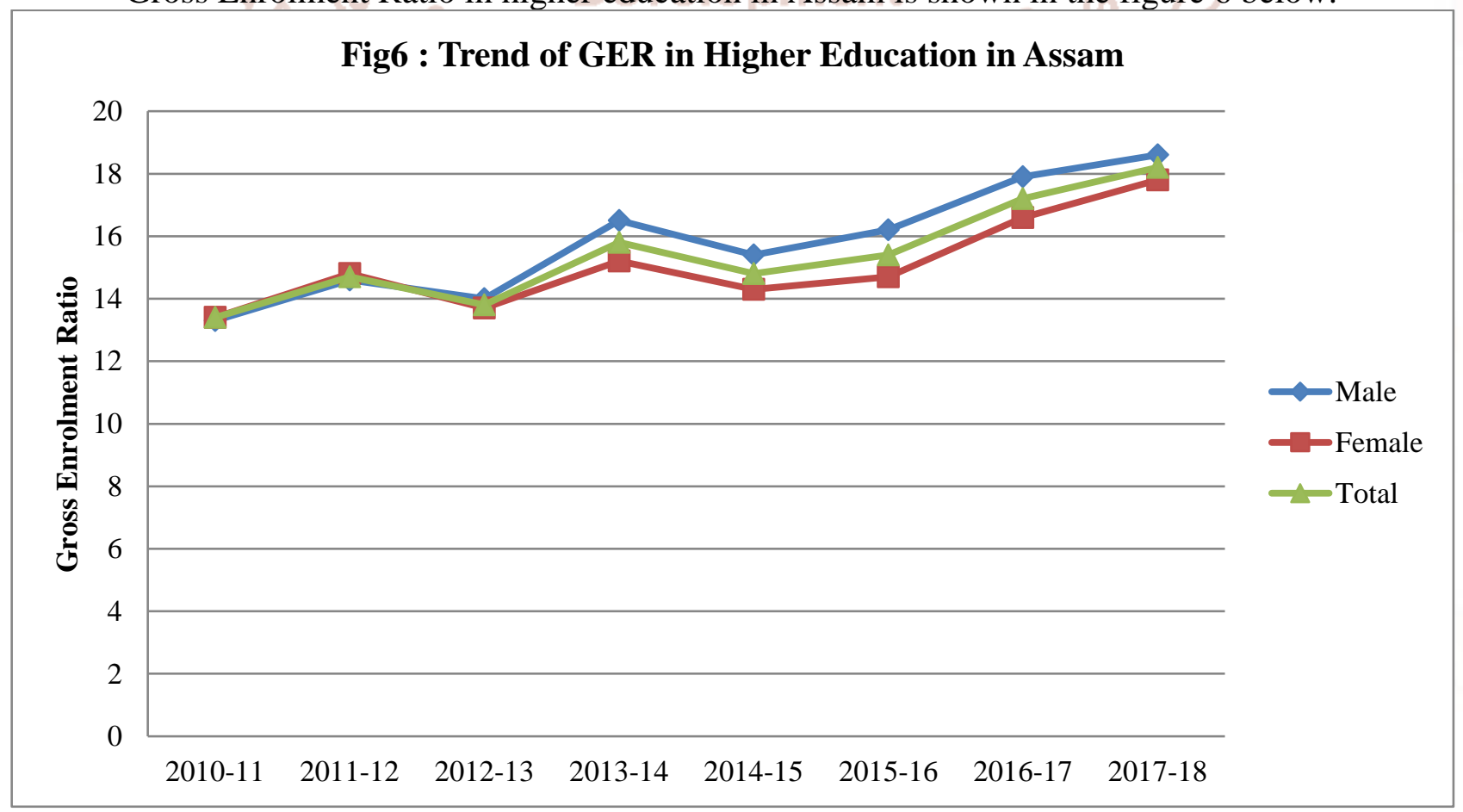

The figure 6 shows that for the beginning three years there was not much difference between GER of male and female and total GER is equal to female GER. But after 2012-13, GER of both male and female shows a fluctuating trend. If we compare gender wise GER we see that GER of male is higher than GER of female. 


\subsubsection{Gender Parity Index:}

Education is one of the vital factor of ensuring gender equality and empowerment. The Gender Parity Index (GPI) is the ratio of the number of female students enrolled at different level of education to the corresponding number of male student in each level. GPI is the ratio of the female to male values of GER. A Gender Parity Index of 1 indicates parity between sexes (MHRD, 2011). The trend of GPI in India and Assam is shown in the figure 7.

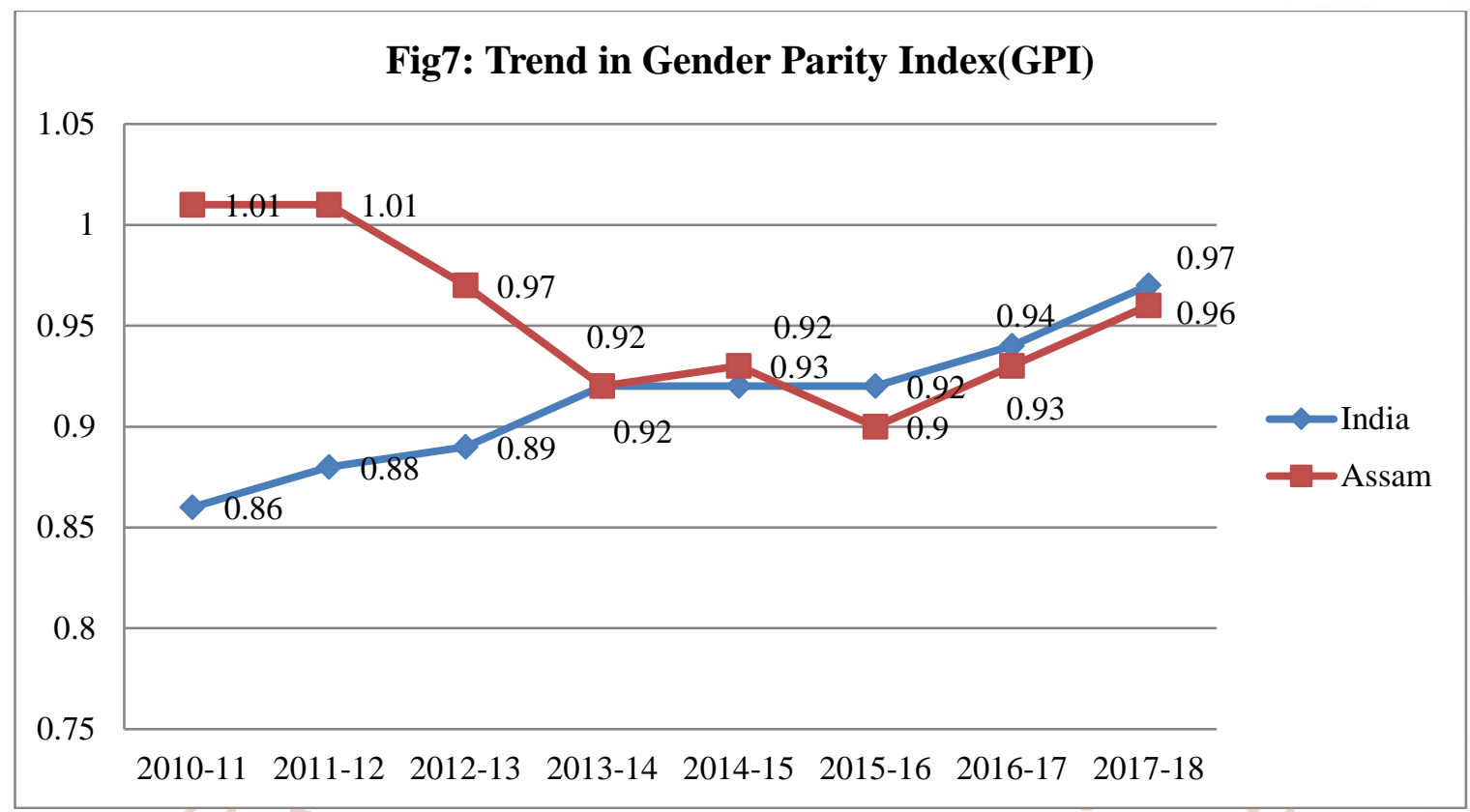

\section{Source: All India Survey of Higher Education, MHRD}

It is seen from the figure 7 that during 2010-11 to 2012-13 there was a wide gap between the GPI of Assam and India as a whole. In 2013-14 GPI of Assam is equal to all India level. After 2014-15, national GPI has increased in comparison to the GPI of Assam. Figure shows that in 2010-11 and in 201112 , the enrolment in higher education is favourable to females in Assam as the corresponding GPI has crossed the limit 1. But, after that the GPI has decreased and the enrolment in higher education became unfavourable to female. At national level, for the whole years the GPI is less than one. It means there is no parity between male and female regarding enrolment in higher education.

\subsection{Conclusion:}

Higher education plays an important role in the creation of human capital. After independence India made significant improvement in the field of education including higher education. Growth of higher educational institution as well as enrolment in higher education has increased during the postindependence era. Government expenditure on higher education increased tremendously and it helped in the expansion of this sector. But, despite the massive increase in number of higher educational institution, the percentage of enrolment is not very high. Because, higher education is still not accessible to the poorest section of the population due to high cost associated with it. Again, from the above analysis we found that lack of equity in higher education is another problem. Therefore quality and equity dimensions of higher education need serious attention. Government should take proper steps to remove all the obstacles present in the growth path of higher education and for this, liberal funding by the government is very much necessary.

\section{References:}

1. Chakrabarti, A \& Joglekar, R. (2006). Determinants of Expenditure on Education: An Empirical Analysis using state Level Data. Economic and Political weekly, 41(15), pp 14651472

2. Best, John W. and Kahn, James V. Research in Education, Prentice- Hall of India, New Delhi-01, 2004

3. Chand S.N (2013). Public Finance. New Delhi: Atlantic Publishers and Distributors (P) LTD 
4. Garret, E. Hanry and R. S. Woodworth Statistics in Psychology and Education, Vakils, Feffer and Simons Ltd, Bomby-38, 1981

5. Koul, Lokesh, Methodology of Educational Research, Vikas Publishing House Ltd., New Delhi-14

6. Mitra, Anuneeta. (2015). Public Spending in Higher Education in India : A Benefit Incidence Analysis. Higher Education for the Future. Vol.2, Issue 1, pp- 71-91

7. Pandey $\mathrm{T}$ (2016). Imperial Journal of Interdisciplinary Research, Vol 2, Issue 5, pp 1428-1435

8. Tasleem Araf C (2016). Trends, Growth and Changing Patterns of Public Expenditure on Education in India. Journal of Global Economics, Vol 4, Issue 4
Government Reports:

1. All India Survey on Higher Education 2016-17, Ministry of Human Resource Development, Government of India, New Delhi 2017

2. All India Survey on Higher Education 2017-18, Ministry of Human Resource Development, Government of India, New Delhi 2018

3. An Analysis of Budgeted Expenditure on Education 2011-12 to 2013-14. Ministry of Human Resource Development, Government of India

4. Report of Justice Dr. K. Punnaya Committee on University Grant Commission funding of Institutions of Higher Education (1993)

5. State Finances. A Study of Budgets of 2017-18 and 2018-19, Reserve Bank of India, July 2018

6. Statistics of Higher and Technical Education 2007-08 published by Government of India, MHRD, Bureau of Planning, Monitoring and Statistics, New Delhi, 2011 